\title{
Technological standardization with and without borders in an interacting agents model
}

Citation for published version (APA):

Cowan, R., \& Cowan, W. (1998). Technological standardization with and without borders in an interacting agents model. MERIT, Maastricht Economic Research Institute on Innovation and Technology. MERIT Research Memoranda No. 015 https://doi.org/10.26481/umamer.1998015

Document status and date:

Published: 01/01/1998

DOI:

10.26481/umamer.1998015

Document Version:

Publisher's PDF, also known as Version of record

\section{Please check the document version of this publication:}

- A submitted manuscript is the version of the article upon submission and before peer-review. There can be important differences between the submitted version and the official published version of record.

People interested in the research are advised to contact the author for the final version of the publication, or visit the DOI to the publisher's website.

- The final author version and the galley proof are versions of the publication after peer review.

- The final published version features the final layout of the paper including the volume, issue and page numbers.

Link to publication

\footnotetext{
General rights rights.

- You may freely distribute the URL identifying the publication in the public portal. please follow below link for the End User Agreement:

www.umlib.nl/taverne-license

Take down policy

If you believe that this document breaches copyright please contact us at:

repository@maastrichtuniversity.nl

providing details and we will investigate your claim.
}

Copyright and moral rights for the publications made accessible in the public portal are retained by the authors and/or other copyright owners and it is a condition of accessing publications that users recognise and abide by the legal requirements associated with these

- Users may download and print one copy of any publication from the public portal for the purpose of private study or research.

- You may not further distribute the material or use it for any profit-making activity or commercial gain

If the publication is distributed under the terms of Article $25 \mathrm{fa}$ of the Dutch Copyright Act, indicated by the "Taverne" license above, 


\title{
Technological Standardization
}

\section{with and without Borders in an Interacting Agents Model}

\author{
Robin Cowan*, William Cowan** \\ *University of Western Ontario, Canada and University of Maastricht, The Nether- \\ lands \\ **University of Waterloo, Canada
}

\begin{abstract}
This paper models an economy in which a large number of agents are choosing among several technologies. There is a single, global, market for the technologies but they are subject to localized network externalities in use. We examine the issue of technological standardization, and the coordination of choices among the agents. We generate equilibirum conditions under two spatial conditions - agents located in a featureless plain, and agents located in pre-existing regions with strong borders. We find the conditions under which standardization exists - globally, only locally, or not at all — and derive the effects of different parameters. The presence of a phase change is demonstrated. An appendix provides a derivation of the equilibrium condition used in many interacting agents models.
\end{abstract}

Keywords: Technogical Standardization, Interdependence, Interacting Agents Externalities J.E.L.Codes:

This paper is a heavily revised version of Cowan and Cowan (1994). We acknowledge helpful discussions with Philip Gunby, and Staffan Hultén and Peter Swann, none of whom is responsible for any remaining errors. 


\section{Introduction}

Many economic phenomena share the feature that agents interact directly, in ways not mediated by the market. Language acquisition, location decisions, the adherence or lack thereof to conventions, belief formation, are all situations in which agents' actions have externalities. The value to an agent of learning or retaining a language depends heavily on whether other agents do the same; location decisions and neighbourhood formation are well-known to be subject to agglomeration economies and sometimes dis-economies; similarly, the benefits of adherence to conventions and the ease of belief formation are affected by whether others are conforming or others have those beliefs.

This paper is a contribution to the literature on interacting agents, motivated by another phenomenon having similar characteristics, namely technological standardization. The literature on this subject is one in which the idea of non-market, direct interactions among agents is common. ${ }^{1} \quad$ Standard models of technological choice or standardization assume that there are positive externalities to adoption, stemming from learning effects, (Arthur (1989), David (1985)) or network externalities (Farrell and Saloner (1985), Katz and Shapiro (1985)). In the early models, these externalities were global, in the sense that the choice of one agent affects the net benefits to every other agent in the economy making the same choice. One of the standard results from these models is that bandwagons can form and that, typically, total standardization occursevery agent uses the same technology. ${ }^{2}$ Recently, though, economists have begun to show interest in the issue of localized externalities. This follows the observation that network externalities, broadly defined, are often localized - one shares much more information about computer software for example with co-workers in nearby offices than with agents at the other end of the country. Even the proto-typical network technology - the telephone - has externalities that are localized to the people an agent is likely to call (friends and colleagues by and large). Thus the positive externalities an agent receives from the adoption decision of another agent will depend upon who, or where that other agent is. ${ }^{3} \quad$ Part of the concern is that the results of the earlier models often seem to be too strong, since we do observe partial standardization: cases in which two or more incompatible technologies co-exist. Further, there is a problem

1 For a survey of the recent interacting agents literature, see Kirman (1997). See David and Greenstein (1990) or Foray (1989) for surveys of the literature on technology choice and standardization.

2 See Bassanini and Dosi for recent results that indicate the possibility of variety under conditions similar to those of the early technology choice models.

3 Assumptions of local externalities also exist outside the field of technology choice: Durlauf (1993), uses local interactions in a model of macro-economic behaviour over time; Glaeser et al. (1996) model criminal behaviour using local externalities in choices. 
in principle with models that examine only global externalities. This is that they can say little, if anything, about the spatial structure of technology use. Spatial patterns in economic activity arise through local interactions, and these are missing in the early models of technology choice.

Because part of the concern in this literature is with the efficiency implications of technology competitions it is important to understand situations of partial standardization. Total standardization appears to be efficient in a static sense in that all possible positive externalities are captured. ${ }^{4}$ Benefits from learning effects, network externalities, or thick markets for peripheral goods, which constitute the classical sources of increasing returns to adoption, all imply that social costs decrease with the extent of standardization. On the other hand, some economists have expressed concern that this type of static efficiency conflicts with dynamic efficiency. Innovation, because it builds on current knowledge, is facilitated when there is a wide variety of activity in the economy. Standardization reduces that sort of variety, which would be bad for innovation, and so for dynamic efficiency. ${ }^{5}$ Thus David et al. (1998) study the locational features of technology use in a percolation model in which competing technologies operate under local increasing returns to adoption. In their simulation, neighbourhoods of users form in which all agents use the same technology. Theoretically, these states may be meta-stable, though, in that random perturbations will eventually take the economy to lower energy rest state in which total standardization takes place. Their model also includes switching costs, which an agent must incur if he changes from one technology to another, and which thus act to freeze the economy in these meta-stable states. An and Kiefer (1995) model the technology choice problem as a birth and death process in which technology use is subject to local externalities. They find that co-existence of technologies is only possible if agents are living in a high $(\geq 3)$ dimensional space, but can say nothing about the spatial nature of that co-existence.

The present paper combines the structures of the earlier and the recent work, and develops a model in which both local and global externalities are present. This modelling innovation changes the nature of the results, so that under a range of parameter values in equilibrium more than one technology is present. ${ }^{6}$ In fact, in equilibrium

\footnotetext{
4 Implicit here, and present in most of the early models, is the assumption that the positive externalities are unbounded and that no congestion effects exist. In the present paper our assumptions about the nature of externalities can be interpreted as representing certain kinds of congestion effects. In Cowan and Cowan (1998) we include congestion explicitly in a model of R\&D location.

5 See Metcalfe (1998), or Revue d'Economie Industrielle (1992).

${ }^{6}$ Kiefer et al. (1991) use results from the literature on Ising models to examine binary choices in one and two dimension lattice spaces. They investigate the presence of equilibrium,
} 
variety and standardization co-exist in a stable, (as opposed to meta-stable), state. We are also able to use the model we develop to begin to examine the spatial nature of standardization, and the formation of patterns is technology use.

The recent work on interacting agents models relies heavily, for the derivation of results, on the Gibbs or Boltzmann distribution. This distribution describes the probability of observing a system or a particle, depending on the application, in a particular state. In the economics literature the foundations of the distribution are seldom discussed, however. For example, in his introduction to the literature on chaos and finacial markets, Brock notes that "the probability structures put forth in Section 1 [sic] of our paper may appear arbitrary and chosen merely for convenience." (Brock, 1993, p. 49). He follows this comment with a nice justification of the assumption that leans on the fact that it is consistent with entropy maximiztion. The present paper includes an appendix in which we provide a derivation, developed in the context of technology choice, of this distribution of states in interacting agents models.

It is worth pointing out that in special cases it is possible to generate this equilibrium distribution from micro-economic discrete choice random utility models, but to do so requires strong distributional assumptions. In particular, the random component of utility must be distributed as a double exponential (Yellot, 1977; see also Anderson et al. (1992), and footnote 7 below, or Durlauf's (1997) survey.) The derivation we provide, though, by assuming a large number of agents, finesses this assumption - the standard logistic distribution comes from the aggregation of the actions of many agents. In the appendix we use our derivation to cast light on what is being included in making this assumption.

\section{The Model}

There are $N$ agents, indexed by $n$, and $Q$ technologies, indexed by $q . N$ is large but finite. Agents are at fixed locations in space and adopt technologies in order to perform on-going tasks. An agent adopts a single technology by acquiring a capital good embodying the technology chosen. Capital goods have finite but random lifetime, and when an agent's capital good wears out, the agent must re-choose, and adopt another (possibly the same) technology.

For each technology, there is a single, competitive market for capital goods, so every agent faces the same supply curve for the good. Without loss of generality, we assume

and the amount of entropy in it, as related to parameter values. Their concern with spatial issues is limited to correlation distances, however. 
that the supply curve for each good is identical. We make conventional assumptions about the production of capital goods, namely marginal cost pricing, decreasing returns in production, and therefore upward sloping supply curves. The supply curve for a good embodying technology $q$ is given by $P_{q}=f\left(Z_{q}\right)$ where $Z_{q}$ is the quantity supplied. We assume a fixed population of agents and that this market always clears, so $Z_{q}$ is interpreted as the global market share of technology $q$. This supply curve determines the cost of adopting technology $q$.

Benefits to adopting a technology are affected by two things. First, there is an effect idiosyncratic to the adopter, stemming from the fact that different technologies are differently suited to different tasks. The tasks performed by an agent differ both across time and across agents, so at any point in time, the technology employed by an agent might be well or badly suited to the task he is performing. Second, there are localized complementarities in use - the benefit from using a technology increases as more of an agent's nearby neighbours use that technology. A good example of this effect is the informal transmission of information about micro-computer hardware and software among people in nearby offices. The value of the benefits to agent $n$ from adopting technology $q$ are $B_{n, q}=g\left(z_{n, q}\right)+h_{n, q}$, where $z_{n, q}$ is the proportion of nearby agents using technology $q$, and $h_{n, q}$ is the inherent value of the technology in the task to which agent $n$ will put it. ${ }^{7}$

We assume that agents are non-strategic and myopic. This is a standard assumption made in these models, as it is in related models in evolutionary game theory, often on the grounds of tractability. ${ }^{8} \quad$ An assumption of rational expectations on the part of the agent would be unreasonable here, since the agent would be faced with a serious computation problem. Thus part of the agenda in work using models of this sort is an examination of conditions under which myopic agents can find a steady state from which the system does not move, in which expectations are consistent with the state of the world. The latter is embedded in the condition, also typically imposed, that the probability of an agent performing an action is equal to the proportion of agents doing it.

At each moment of choice, then, the agent's problem is to maximize net benefits from adoption:

$$
\max _{q \in\{1 \ldots Q\}} g\left(z_{n, q}\right)+h_{n, q}-f\left(Z_{q}\right) .
$$

\footnotetext{
7 "Nearby" will be defined precisely below.

8 For a general discussion on the appropriateness of assumptions of bounded rationality, see Conslick (1996).
} 
A remark is needed here about the idiosyncratic effect. From the point of view of the agent, the idiosyncratic effect is well-known. He knows which task he is performing at the moment of choice. Thus his problem is completely deterministic. The analyst has a different point of view however. We have assumed that there are a large number of agents. It would be unreasonable to think that the analyst could have information about the tasks of each of them. Thus from the analyst's point of view, for any particular agent, the idiosyncratic effect is random. ${ }^{9}$ Thus we will treat $h_{n, q}$ as a random variable, and without loss of generality, assume it has a mean of $0 .{ }^{10}$ This treatment allows agents' decisions to be aggregated to generate results about the prevalence of each of the technologies in the economy.

The model is a member of the class of interacting agents models, which have been taken up recently in the economics literature, and have been analyzed using the techniques of statistical mechanics. ${ }^{11}$ These models bear a close relation to discrete choice, random utility models in that the object of both types of analysis is the probability that an agent performs a given action, (and in models of many agents, the proportion of agents performing it) rather than the identification of which action an agent (surely) performs. Durlauf (1997) reviews this literature, and in the next section we will use some of the results he presents. It is worth pointing out, however, that in Durlauf's review he identifies two types of models - those in which interactions are local, and those in which interactions are global. The current model contains both local and global interactions, and this innovation has interesting effects on the results.

Statistical mechanics type models have been employed to analyse a wide variety of systems in different disciplines, and there are several approaches to the derivation of equilibrium conditions. The equilibrium condition is typically expressed as the Boltzman distribution, which has been derived in many ways. ${ }^{12}$ The probability of observing

\footnotetext{
9 See Anderson, de Palma and Thisse (1992), pp. 31-33 for a discussion of this approach to including randomness as part of the analysis in random utility models.

10 Yellott (1977) shows that for a random utility model of a single agent, $h$ being distributed as a double exponential is necessary and sufficient to generate a logistic probability of performing an action. Derivation of the equilibrium distribution in a model with many agents finesses this assumption (see the appendix). The logistic function that emerges arises from the aggregation of many agents to generate results on average behaviour. In effect it comes out of the combinatorial aspect of there being many ways in which a large number of agents can act and produce the same average behaviour.

11 The earliest exemplar in economics is Föllmer (1974).

12 Schroedinger(1952), Pathria (1972) and Waldram (1985) provide but three distinct derivations. The appendix contains a derivation that is developed specifically in the technology choice context.
} 
an agent in a particular state $x$ given by

$$
\operatorname{Pr}(x)=\frac{\exp \{-\beta B(x)\}}{\sum_{x^{\prime}} \exp \left\{-\beta B\left(x^{\prime}\right)\right\}},
$$

where the sum in the denominator is over all possible values of $x$, and where $B(x)$ is the net benefit to the agent of being in state $x$. This distribution is the familiar logistic distribution seen in discrete choice, random utility models, and is also known as Luce's Choice Axiom. The parameter $\beta$ measures the inverse of the strength of agent heterogeneity. In our model, agent heterogeneity arises because agents perform different tasks. Thus $\beta$ measures the extent to which tasks differ across agents and across time. The important measure of difference here relates to how differently suited are the technologies to the different tasks.

One result that is clear from the development of the literature to date is that the nature of the equilibrium depends on the nature of the interactions among agents. In general, the way agents interact will be affected by the nature of the space in which they are located. The ease with which, and thus the extent to which, agents can communicate with each other is central to the types of interactions they have. This will be determined, in part at least, by the nature of the space in which they are located. In a space that has natural boundaries, mountain ranges perhaps, communication patterns will be different from those in one that does not. We examine two extreme topologies: one in which agents exist on a featureless plain; and the other in which agents exist in a world with pre-defined boundaries between regions.

\section{Homogeneous space}

We first consider agents located on a featureless plain.

In a metric space many interactions are translationally symmetric: they depend only on the vector joining the two agents, not on the absolute position of the agents. Such spaces are called homogeneous, and interactions depending solely on the distance between agents are the most common type in a homogeneous space.

Define the distance, $d_{m n}$, between two agents $m$ and $n$, as the euclidean distance between their locations in a two-dimensional space. To avoid boundary problems, we assume that the space is toroidal. To agent $n$, the benefit from using the same technology as agent $m$ is inversely related to the distance between them. In this case, $z_{n, q}$ is no

longer a simple proportion but rather a weighted average: $z_{n, q}=\sum_{m=1}^{N} S_{m, q} G\left(d_{m n}\right)$, where $\left|G^{\prime}(d)\right| \leq 0$ for all $d$, and $S_{m, q}$ has the value of 1 if agent $m$ is using technology $q$, and 0 otherwise. If $G(\cdot)$ is defined appropriately then $g(z)$, the benefits arising from 
externalities from other agents, can be the identity function. We have assumed that the local externality is positive, so $G(d)>0$.

In general, equilibria are found by solving a state equation, which in this model is

$$
s_{n, q}=\frac{\exp \left\{-\beta\left(\sum_{m} G\left(d_{n m}\right) s_{m, q}-f\left(\sum_{m} s_{m, q}\right)\right)\right\}}{\sum_{p} \exp \left\{-\beta\left(\sum_{m} G\left(d_{n m}\right) s_{m, p}-f\left(\sum_{m} s_{m, p}\right)\right)\right\}} .
$$

Here $s_{n, q}$ is the probability that agent $n$ uses technology $q G(\cdot)$ captures externalities that are distance dependent, and $f(\cdot)$ captures externalities that are generated by population averages. The variable $\beta$ measures the inverse of the strength of agent heterogeneity, and idiosyncratic effects; $\beta$ is large when inter-agent variation of idiosyncratic effects is small and small when the variation is large.

If we restrict our attention to binary choices, and if $f(\cdot)$ is linear, equation 2 can be re-written as a hyperbolic tangent.

$$
x_{n}=\tanh \left[\beta / 2\left(\sum_{m} G\left(d_{m n}\right) x_{m}-f\left(\sum_{m} x_{m}\right)\right)\right],
$$

where $x_{n}$ is the difference between the probability that agent $n$ uses technology one and the probability that agent $n$ uses technology 2 .

Brock and Durlauf (1995) demonstrate several results in a slightly less general case then the one we have described, but their results carry over, since the form of the state equation is the same. These results are presented as Theorem 1 in Brock and Durlauf (1995), or Theorem 1 in Durlauf (1997).

Briefly, there are two possible regimes in this system. If agent heterogeneity is high relative to the strength of the local interactions, ( $\beta$ is small relative to $G^{\prime}$ ), then there is a single equilibrium, with $x=1 / 2$. That is, because agents' tasks vary considerably one from the other, and because different technologies are differently suited to different tasks, agents are unable to coordinate to capture externalities in adoption. Thus there is no standardization. If agent heterogeneity falls, or the strength of the local interaction effect increases, however, there are benefits from standardization that exceed the benefits from trying to adopt the technology best-suited to the task being performed at the time, and at least some degree of standardization occurs. In general, which technology dominates is indeterminate. Thus in the regime in which standardization occurs, multiple equilibria exist. 
Many different functional forms for $f(\cdot)$ and $G(\cdot)$ have been investigated in other disciplines because they are considered to describe a variety of ordering processes in nonuniform systems. Examples include patterns in animal coats (Turing 1952), patterns in neural connections in the primate visual cortex (Thompson et al. 1992), or patterns of condensation in the presence of emulsifying agents (Elder and Grant 1990). These analyses show several common qualitative features of the spatial distributions of activity that are likely to occur for competing technologies, regardless of the precise functional forms of the interaction mechanisms, provided local externalities are positive, and global externalities negative.

There are generally four types of equilibria: three with structure, and one without. Equilibria without spatial structure exist when the effects of agent heterogeneity are large. In this case, every agent is governed by idiosyncratic effects and chooses a technology independently of his or her neighbours' choices. By contrast, structured equilibria all occur when the strength of agent-dependent idiosyncratic effects is small. The first structured equilibrium is uniform - in which every agent uses the same technology. This occurs when there is a relatively strong external force encouraging the use of a particular technology. This can be modelled as a situation in which $h_{n, q}$ has a non-zero mean for some $q$ and all $n$. In the second and third structured equilibria the market is not standardized on a single technology. Spatially, the market is divided into areas in which all agents use one technology, different areas employing different technologies. In the second equilibrium, these areas resemble stripes. It occurs when there is no net preference for one technology over another, ( $h_{n, q}$ has zero mean), or when the net preference is small. The third structured equilibrium does not occur in all systems. It coincides with amounts of net preference that are intermediate between the amounts that produce uniform and striped equilibria. In this equilibrium, the spatial segmentation of the market resembles spots: islands of Macintosh users in a sea of Windows machines. In the striped and spotted equilibria, the stripes and spots have characteristic sizes, with all stripes being approximately the same width, and all spots being approximately the same diameter. These characteristic sizes depend on parameter values, and in particular on the tension between the degree of agent heterogeneity and the strength, and extension of local positive feedbacks.

\section{Heterogeneous space}

While the evolution of the homogeneous space model will create a division of the space into regions, in many economic contexts natural regions exist prior to any techno-

logical competition. For example, an economy can be divided into groups of agents all of whom have many interactions with each other, but few interactions with agents outside 
the group. Agents within a country in Europe, within a firm, or within a department of a university, say, form a natural group within which interaction is much stronger than it is with agents outside the group. Local adoption externalities operate through interactions of nearby agents, so a firm would represent a relatively closed neighbourhood of agents. In this section we assume that agents operate within a neighbourhood or region of nearby agents. Within a region all agents interact directly with each other, and distance within a region is not important. The regions are closed, though, in the sense that agents within one region have no direct interactions with agents who are in other regions. ${ }^{13}$ We develop the technique for $R$ regions, and $Q$ technologies, but for simplicity in presentation we use as an example the two-region, two-technology case, which exhibits generalizable qualitative and quantitative behaviour.

\subsection{Equilibrium Conditions}

Assume there are $R$ regions, indexed by $r$, and $Q$ technologies, indexed by $q$. Region $r$ has $N_{r}$ agents. Define $n_{r}$ as $N_{r} / N$, the proportion of agents in region $r$. In this model distance, as defined in the homogeneous space model, is not a factor. ${ }^{14}$ The benefit to agent $n$ from having the same technology as agent $m$ depends only on whether or not the two agents are in the same region. Thus $z_{n, q}$, the proportion of agents 'near' to $n$ using technology $q$, is the same for every member of a region, so we can refer to $Z_{r, q}$, the proportion of agents in region $r$ who use technology $q$. In this model, equilibrium in the macro state is described by an $R$ by $Q$ matrix of proportions of technology use.

As above, there is a global market for the captial good with the price determined by $P_{q}=f\left(Z_{q}\right)$, and benefits arising from externalities, $g\left(Z_{r, q}\right)$. We assume that $f(\cdot)$ and $g(\cdot)$ are linear, $f\left(Z_{q}\right)=W Z_{q}$ and $g\left(Z_{r, q}\right)=V Z_{r, q}$ with $W$ and $V$ positive constants. Within region $r$, the equilibrium proportion of users using technology $q$ is written as

$$
Z_{r, q}=\frac{e^{\beta\left(V Z_{r, q}-W Z_{q}\right)}}{\sum_{p=1}^{Q} e^{\beta\left(V Z_{r, p}-W Z_{p}\right)}}
$$

where $Z_{q}=\sum_{r=1}^{R} n_{r} Z_{r, q}$. Restricting attention to the case of two technologies, it is convenient to use a change of variables, and to pursue the analysis in terms of the

\footnotetext{
13 The spatial structure in this model is also examined by Brock (1993) in an appendix.

14 Another interpretation is that the economy is divided into groups of agents defined by their interactions. Within a group, all agents interact to the same extent (are equal distance from each other). But any two agents from different groups never interact directly (are of infinite distance from each other). This interpretation suggests that the general model is very flexible, but that the definition of the distance metric is likely to be very important. This interpretation also indicates that the homogeneous and heterogeneous space cases are two extremes of the possible topologies of interaction.
} 
differences in the extent to which the technologies are adopted within a region. ${ }^{15}$ Thus we define $x_{r}=Z_{r, 1}-Z_{r, 2}$, so that $x_{r}$ is the difference between the proportions of agents using technologies one and two in region $r$. This allows us to write

$$
x_{r}=\tanh \left(\beta / 2\left(V x_{r}-W \sum_{r=1}^{R} x_{r} n_{r}\right)\right) .
$$

In the case of two equal-sized regions, equation (5) becomes

$$
\begin{aligned}
& x_{1}=\tanh \left(\beta\left(V x_{1}-W\left(x_{1}+x_{2}\right) / 4\right)\right) \\
& x_{2}=\tanh \left(\beta\left(V x_{2}-W\left(x_{1}+x_{2}\right) / 4\right)\right)
\end{aligned}
$$

and equilibria are defined by the intersections of these two curves.

\subsection{Equilibria}

In general, because of the implicitness of equations 6 and 7 , systems of this sort are very difficult to treat analytically. Some analytical results regarding equilibria and the nature of the comparative statics are available, and numerical solutions indicate the robust presence of other effects. In this section we first prove three propositions about the nature of equilibria in this model. The equilibria exist as solutions to equations 6 and 7 above, which are plotted in Figures 1 and 2 under a variety of parameter values.

Proposition 1: A solution to equations 6 and 7 always exists at $x_{1}=x_{2}=0$.

This is straightforward by substitution, $\operatorname{since} \tanh (0)=0$.

When this solution is stable, which is not always the case, it indicates an equilibrium in which there is no standardization of technology choice. Both technologies appear with the same frequency in both regions. As the next proposition indicates, though, for a range of parameter values solutions implying local standardization with global variety exist.

Proposition 2: If $\beta V>1$ then there exist solutions to equations 6 and 7 of the form $x_{1}=-x_{2}$.

Proof: If $\left(x_{1}^{*}, x_{2}^{*}\right)$ solves equation 6 , then $\left(-x_{1}^{*},-x_{2}^{*}\right)$ is also a solution. The same is true for equation 7 . Further, equation 6 is a reflection of equation 7 around $x_{1}=x_{2}$.

15 Equation (4) is derived in the appendix. 
These properties imply that if equation 6 passes through $x_{1}=-x_{2}$ then equation 7 passes through the same point. Thus we need only show that if $\beta V>1$ equation 6 passes through $x_{1}=-x_{2}$.

Consider $x_{1} \geq 0$. Under equation $6, \lim _{x_{1} \rightarrow 1} x_{2}=-\infty$. Equation 6 passes through the origin and is continuous. Therefore if $d x_{2} / d x_{1}>-1$ at the origin, the curve intersects $x_{1}=-x_{2}$.

$$
\frac{d x_{2}}{d x_{1}}=\frac{1-\operatorname{sech}^{2}\left[\frac{\beta}{2}\left(2 V x_{2}-\frac{W}{2}\left(x_{2}+x_{1}\right)\right]\left[\frac{\beta}{2}\left(2 V-\frac{W}{2}\right)\right]\right.}{\operatorname{sech}^{2}\left[\frac{\beta}{2}\left(2 V x_{2}-\frac{W}{2}\left(x_{2}+x_{1}\right)\right]\left[\frac{\beta}{2}\left(-\frac{W}{2}\right)\right]\right.} .
$$

At the origin $\operatorname{sech}(\cdot)=1$, so we can write

$$
\left.\frac{d x_{2}}{d x_{1}}\right|_{x_{1}=x_{2}=0}=\frac{1-\frac{\beta}{2}\left(2 V-\frac{W}{2}\right)}{\left[\frac{\beta}{2}\left(-\frac{W}{2}\right)\right]},
$$

which is greater than -1 if and only if $\beta V>1$. The same argument applies for $x_{1} \leq 0 . \diamond$

Proposition 2 indicates necessary and sufficient conditions for the existence of equilibria in which standardization within each region takes place, but in which regions standardize on different technologies. Due to the symmetry in the externality functions, and that the supply curves for the two technologies are the same, we observe that both regions standardize to the same extent, though on different technologies. Because the argument applies independently to both $x_{1} \leq 0$ and $x_{1} \geq 0$, there are two possible equilibria. Regions always standardize on different technologies, but which technology dominates in each region is not determined.

The next proposition indicates the possibility that both regions standardize on the same technology.

Proposition 3: If $\beta(V-W / 2)>1$ then solutions to equations 6 and 7 exist at $x_{1}=x_{2}$.

Proof: By the arguments given under proposition 2, we need only show that equation 6 intersects $x_{1}=x_{2}$. Since $\lim _{x_{1} \rightarrow 1} x_{2}=-\infty$, this occurs if $d x_{2} / d x_{1}>1$ at the origin. Noting again that $\operatorname{sech}(0)=1$, we write

$$
\frac{d x_{2}}{d x_{1}}=\frac{1-\operatorname{sech}^{2}\left[\frac{\beta}{2}\left(2 V x_{2}-\frac{W}{2}\left(x_{2}+x_{1}\right)\right]\left[\frac{\beta}{2}\left(2 V-\frac{W}{2}\right)\right]\right.}{\operatorname{sech}^{2}\left[\frac{\beta}{2}\left(2 V x_{2}-\frac{W}{2}\left(x_{2}+x_{1}\right)\right]\left[\frac{\beta}{2}\left(-\frac{W}{2}\right)\right]\right.}>1 .
$$

This reduces to $\beta(V-W / 2)>1 . \diamond$

Corollary: It follows immediately from propositions 2 and 3 that since $W>0$, if symmetric equilibria exist $\left(x_{1}=x_{2}\right)$ then equilibria in which $x_{1}=-x_{2}$ also exist. 
These propositions indicate a phase change in the behaviour of the model. Below the critical value of $\beta V$, there is a unique equilibrium at $x_{1}=x_{2}=0$. This implies that in both regions each technology has a market share of $1 / 2$. In other words, there is no standardization either at the local or global level. When $\beta V$ is greater than one, $x_{r}$ moves away from zero, indicating that local standardization takes place. In the regime where $\beta V>1$ but $\beta(V-W / 2)<1$, (a case covered by Proposition 2), global variety exists, even in the face of local standardization. When $\beta(V-W / 2)>1$, however, equilibria exist in which global standardization takes place, and variety is lost if the system rests in one of these equilibria. In any non-zero equilibrium, however, both regions standardize to the same extent.

\subsection{Stability}

For certain regions of the parameter space, this system has multiple solutions-3, 5 or 9 depending on parameter values, the number increasing as $\beta(V-W / 2)$ increases. Not all solutions are stable, however, and the stability of a solution depends on the dynamics around that solution. Analytically, generating stability conditions is extremely difficult except in very special cases. We are able in this model, though, to characterize the solutions so that numerical simulation of the model generates robust results about stability.

There are two types of solutions: $\left|x_{1}\right|=\left|x_{2}\right|$ and $\left|x_{1}\right| \neq\left|x_{2}\right|$. None of the solutions in the class $\left|x_{1}\right| \neq\left|x_{2}\right|$ is stable. This permits us to ignore solutions having $\left|x_{1}\right| \neq\left|x_{2}\right|$ in what follows. Within the first class of solutions, $\left|x_{1}\right|=\left|x_{2}\right|$, if any non-zero solution exists, it is stable, and the solution at the origin is not. When non-zero solutions do not exist, then the solution $x_{1}=x_{2}=0$ is unique and stable.

\subsection{Comparative Statics}

The comparative statics results follow directly from the proofs of the propositions above.

Proposition 4: If $\beta V>1$ then at the equilibria in which $x_{1}=-x_{2}=x, \operatorname{sgn}(\partial x / \partial V)=$ $\operatorname{sgn}(x), \operatorname{sgn}(\partial x / \partial \beta)=\operatorname{sgn}(x)$, and $\partial x / \partial W=0$.

Proof: Equilibria in which $x_{1}=-x_{2}=x$, with $x>0$, must satisfy the condition that $x=\tanh (\beta V x)$. Since $W$ does not appear here, the derivative of $x$ with respect to $W$ will be zero. $\tanh (z)$ is monotonically increasing (decreasing) in $z$ for $z>0$ $(z<0)$. This implies that if $(\beta V)_{1}>(\beta V)_{0}$ then $\left|\tanh \left(x(\beta V)_{1}\right)\right|>\left|\tanh \left(x(\beta V)_{0}\right)\right| \forall x$. $\tanh (\cdot)$ is concave, so as $\beta V$ increases so, in absolute value, does the fixed point of $x=\tanh (\beta V x)$. Since both $\beta$ and $V$ are positive, the proposition follows. $\diamond$ 
Proposition 5: If $\beta(V-W / 2)>1$ then at the equilibria in which $x_{1}=x_{2}, \operatorname{sgn}(\partial x / \partial \beta)=$ $\operatorname{sgn}(x) ; \operatorname{sgn}(\partial x / \partial V)=\operatorname{sgn}(x) ; \operatorname{sgn}(\partial x / \partial W)=-\operatorname{sgn}(x)$.

Proof: At equilibria in which $x_{1}=x_{2}, x$ is determined by $x=\tanh (\beta(V-W / 2) x)$. Given this, the argument is identical to that for Proposition 4, simply noting that in this case $W$ is multiplied by $-1 . \diamond$

These comparative statics results are illustrated in Figures 1 and 2. Figure 1 shows the effects of changing the value of $W$. The value of $W$ falls as we move from panel 1a to panel 1e. Throughout, $\beta V$ is unchanged, but is relatively large. Figure 2 shows the effects of changing the value $\beta V$ with $W$ fixed. It increases moving from panel $2 \mathrm{a}$ to $2 \mathrm{f}$. The two curves in the figure are equations 6 and 7 ; solutions occur at their intersections. The dashed lines show equation 7 and the solid lines show equation 6 .

Propositions 4 and 5 state that as the strength of the local externalities increase, or the degree of heterogeneity decreases (either change increases $\beta V$ ), the extent of local standardization, as measured by the proportion of agents using the dominant technology, increases. Proposition 4 states that the strength of global externalities does not affect the degree of standardization when the economy finds asymmetric equilibria, but proposition 5 states that in symmetric equilibria weakening the global externalities increases the degree of standardization.

The three parameters play different roles in determining the number and nature of equilibria. The interaction of local complementarities and heterogeneity among agents determines whether technologies are distributed uniformly over the space or whether there is a spatial pattern to their use. The interaction between these two parameters also determines how much standardization there is within a region, where the degree of standardization is measured by the proportion of agents using the dominant technology. These results are intuitively appealing in the technology choice context. If nearby agents have very diverse tasks to perform, then in choosing among technologies, they are likely to place more weight on the characteristics of the technologies than on the fact that their neighbours have information about one of them.

The effect of the global decreasing returns to adoption is very different. It plays no role in determining whether standardization occurs. And if the system selects an equilibrium in which different regions standardize on different technologies, it plays no role in determining the degree of standardization within each region. The role of this parameter is thus relatively restricted. If it is weak enough, though, it permits the 
existence of equilibria in which all regions standardize on the same technology; and if that equilibrium is selected, the degree of local standardization increases as the strength of the decreasing returns falls. As the decreasing returns increase in strength, however, the two curves $x_{r}=\tanh \left(\beta\left(V x_{r}-W / 4\left(x_{r}+x_{s}\right)\right)\right.$ approach each other, and in the limit become $x_{1}=-x_{2}$. Thus in the limit, there is a continuum of equilibria, with the defining property that the degree to which technology one is dominant in one region is exactly equal to the degree to which technology two is dominant in the other. This too is intuitively appealing: if $W$ is large, changes in costs of capital goods will dominate other effects, and agents will drive the market to a point at which costs of the two types are equal. This implies equal prominence of the two technologies in the economy as a whole. This can only be achieved if when technology one, say, dominates region one, technology two is exactly equally dominant in region two. ${ }^{16}$

Heterogeneity has two sources - heterogeneity among agents; and heterogeneity within an agent but across time - and it is worth examining in isolation. As noted above, regional separation occurs only if $V>1 / \beta$, where $1 / \beta$ is a measure of heterogeneity. As $\beta$ decreases, then, the system moves to a position where the entire market is uniform in the proportion of agents using any particular technology. A small value of $\beta$ implies that the most important consideration for an agent will be the characteristics of the technologies, and agents will effectively choose on that basis alone, with little consideration of externality effects, which means that there will be no regional differentiation. Thus, in the two-technology model the market shares of each of the technologies is $1 / 2$ (more generally, it is 1 over the number of technologies). As $\beta$ rises, though, there is a first-order discontinuity at $1 / \beta=V$ at which point each region begins to standardize. The degree of standardization (measured as the proportion of agents using the dominant technology in a region) increases rapidly as heterogeneity falls, moving towards total standardization within a region. (When $\beta V=1$ the proportion of agents in any region using the dominant technology is $1 / 2$. Ninety-five percent use the dominant technology when $\beta V=3.25$. When $\beta V=4$ the proportion has risen to about 98 percent.) Thus we can see that the value of $\beta$ will divide a technology choice problem into one of three types: for small values of $\beta$, no standardization occurs; for large values of $\beta$ almost complete regional standardization occurs, and for a small

16 There is a second effect of $W$ that is not strictly within the bounds of the analysis. With reasonable dynamics, as decreasing returns become stronger, and the two curves approach each other, the speed with which the system approaches its equilibrium state will slow down. It will move relatively rapidly to a state in which $x_{1} \approx-x_{2}$, and then move slowly to the equilibrium state in which the two regions are relatively standardized, but on different technologies. This situation is shown in Figure 1, panel a. 
interval of $\beta$ there is partial standardization within regions. This effect is shown in Figure 3.

It is not surprising that the degree of standardization increases as the degree of heterogeneity falls. What is somewhat surprising is the existence of this phase change. For small values of $\beta$ (large degrees of heterogeneity) there is no standardization. But as $\beta$ increases, it passes through a critical value above which the system moves quickly to a state of almost total (local) standardization. This suggests that a critical feature in understanding the existence, or lack thereof, of de facto standardization in particular markets will be understanding the degree and nature of the heterogeneity of the agents involved.

\section{Discussion}

Early models of technology choice included only global increasing returns. The model presented here is a generalization of that model, but we have here presented a model with global decreasing returns. The assumption of global increasing returns can be represented here simply by changing the sign of $W$, the global externality, to make it negative. ${ }^{17}$ When this change is made, several equilibria still exist. If agent heterogeneity is strong, the equilibrium has no spatial structure, as before. As the degree of heterogeneity declines however, structure emerges, and we observe standardization within regions. Again, though, there are two variations of regional standardization. If the global effects are strong, every region standardizes on the same technology. Which technology dominates, of course, is a matter of historical accident. When global effects are weak another type of equilibrium appears, namely one in which different regions standardize on different technologies. (Graphically, a change in the sign of $W$ from positive to negative results in Figures 1 and 2 simply being reflected around the vertical axis.)

A second concern that has been current in technology studies has been the possibility that the market will standardize on an inferior technology. In the model as presented, neither technology can be considered a priori inferior. This can be included, though, simply by subtracting a constant from the costs of using one of the technologies. (This can obviously be generalized to adding different constants to the costs of using each technology, and can also be made region-specific.) In this way, one technology would have an advantage over the others in the sense that, all else equal, it has a lower cost. In general, the discussion of equilibria presented above is maintained, except that

17 This should probably not be interpreted as a downwardly sloping supply curve, at least in general. It should be interpreted to indicate that global positive externalities in consumption outweigh the presence of a upward sloping supply curve. 
there is a tendency for the preferred technology to find a higher degree of adoption than the other technologies. Graphically, this is seen in Figures 1 and 2 as shifting one of the curves away from the origin, which causes a general increase in the probability that that technology is adopted. Again, though, and common to previous work, stable equilibria exist in which the low-cost technology is not the one that becomes standard. Indeed, equilibria exist in which each region standardizes on a technology other than the technology that is lowest cost in that region.

The results above were presented in the case of two regions and two technologies. They generalize in a straightforward way to $R$ regions and $Q$ technologies. Phase changes exist: as $\beta V$ increases the system moves from an equilibrium in which there is no spatial pattern in technology use to one in which regions specialize in a particular technology. It is also the case, as in the formal results presented above, that if the global externality is weak, technologies will not necessarily be equally represented at the global level. To be more precise, the results generalize very directly if there are the same number of regions as there are technologies. If there are more regions than technologies (which seems likely to be a common situation), and if the number of regions is not a multiple of the number of technologies, integer problems will dictate that some technologies are more common at the global level. ${ }^{18}$ Taking into account these integer problems, the results are very robust to this kind of change.

\section{Conclusions}

One feature common to most situations and models of technology choice is that actions of agents, and interactions among them, specified at a micro level, determine the macro structure of the technological system. The relationship between micro interactions and macro structure has been difficult to analyze, and the degree of difficulty increases with the degree of complexity of the interactions. In this paper we have examined a model with both global and local, positive and negative interactions among agents repeatedly choosing among several technologies. There are two particularly striking results. First, when agents are very heterogeneous there is no spatial structure to the market and variety is preserved both locally and globally. As the degree of heterogeneity falls, though, it hits a critical value, below which the market moves very rapidly to a situation with two distinct regions, both of them almost completely standardized-local variety disappears, but variety at the global level is maintained. The second result is that the strength of the global, negative effect has no impact either on whether regional differentiation occurs, or, in most situations, the degree of standardization within either

18 This discussion assumes that regions are the same size. It would have to be modified in obvious ways if regions are of different sizes. 
of the regions. The only role it plays in the model is to determine whether or not an over-standardization equilibrium, in which variety completely disappears, exists.

We observe in many economic situations that interactions among agents are affected by the spatial structures within which agents find themselves. This is true both of spaces that are defined geographically and those that are defined by some other form of proximity. One of the conclusions of this paper is that this observation indicates a route through which variety in technology use can be preserved. Borders between regions, which may exist for reasons independent of the technology, provide, for some agents, insulation from the agglomerating forces that would otherwise be felt from the actions of other agents. Stable, as opposed to meta-stable, equilibria exist in which variety is preserved, even if the technologies are identical in all respects. Variety is only guaranteed, though, if there is some form of interaction between the regions, and this interaction is not agglomerative in effect. It seems natural to think of regions as nations, but often this is not the scale at which these forces act. Very often the types of interactions that contain agglomerative effects have a very short length scale; regions may often be more appropriately thought of as much smaller, on the scale of the size of a firm, or perhaps productive unit. The definition, and therefore the size of a region will in general, though, be determined by the nature of the interactions among agents.

We have employed a technique that enables us to take a specific micro-economic problem, in a model with heterogeneous agents and complex, spatially dependent interactions among them, and generate results about the macro state of the economy. While we have employed this technique to examine a very simple technology choice model, it can easily be extended to more complex models. It is within the structure of the technique to allow regions containing different numbers of agents; it is also relatively straightforward to introduce regional biases in the choice process, stemming, for example, from the procurement policies of a firm or a government which favour a particular technology. A more difficult problem, but one that can be attacked using this framework, is the problem of fuzzy borders between regions. We have assumed that no agents in a region interact with agents in other regions. This is an extreme case, and there are common examples in which there is some interaction across regions, though noticeably less than within regions. A modification of the modelling technique we have employed here can be used to address this issue. Finally, though we have been concerned with technology choice and standardization, the techniques we have developed can be applied to other economic contexts in which identifiable, complicated interactions among many agents determine macro-economic structure. 
Figure 1

Showing the effects of decreasing the strength of the global externality.
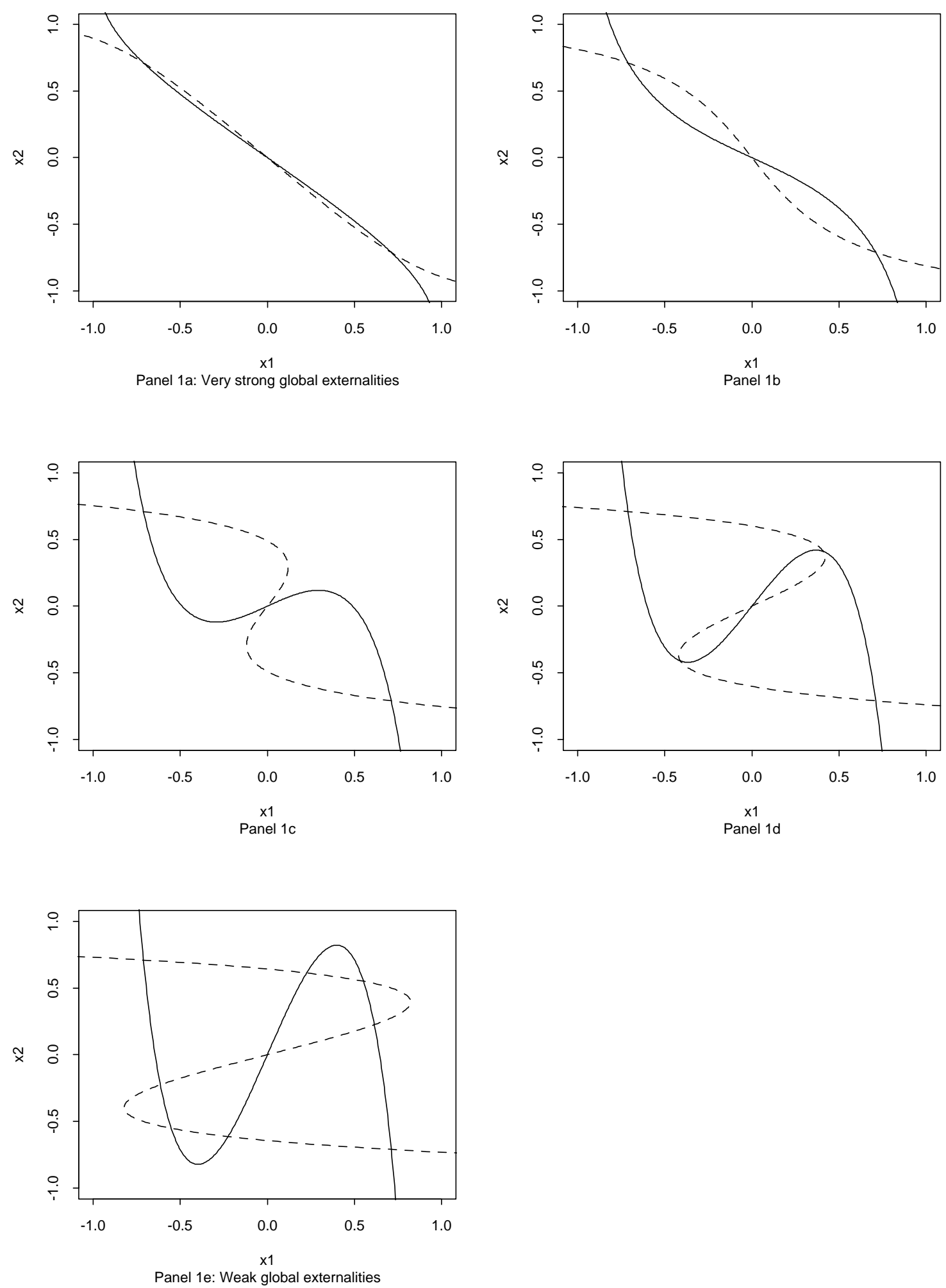

Equilibria exist at the intersections of the two curves.

The dashed curve is equation 6 ; the solid curve equation 7 . 
Figure 2

Showing the effects of increasing the strength of local externalities relative to agent heterogeneity.
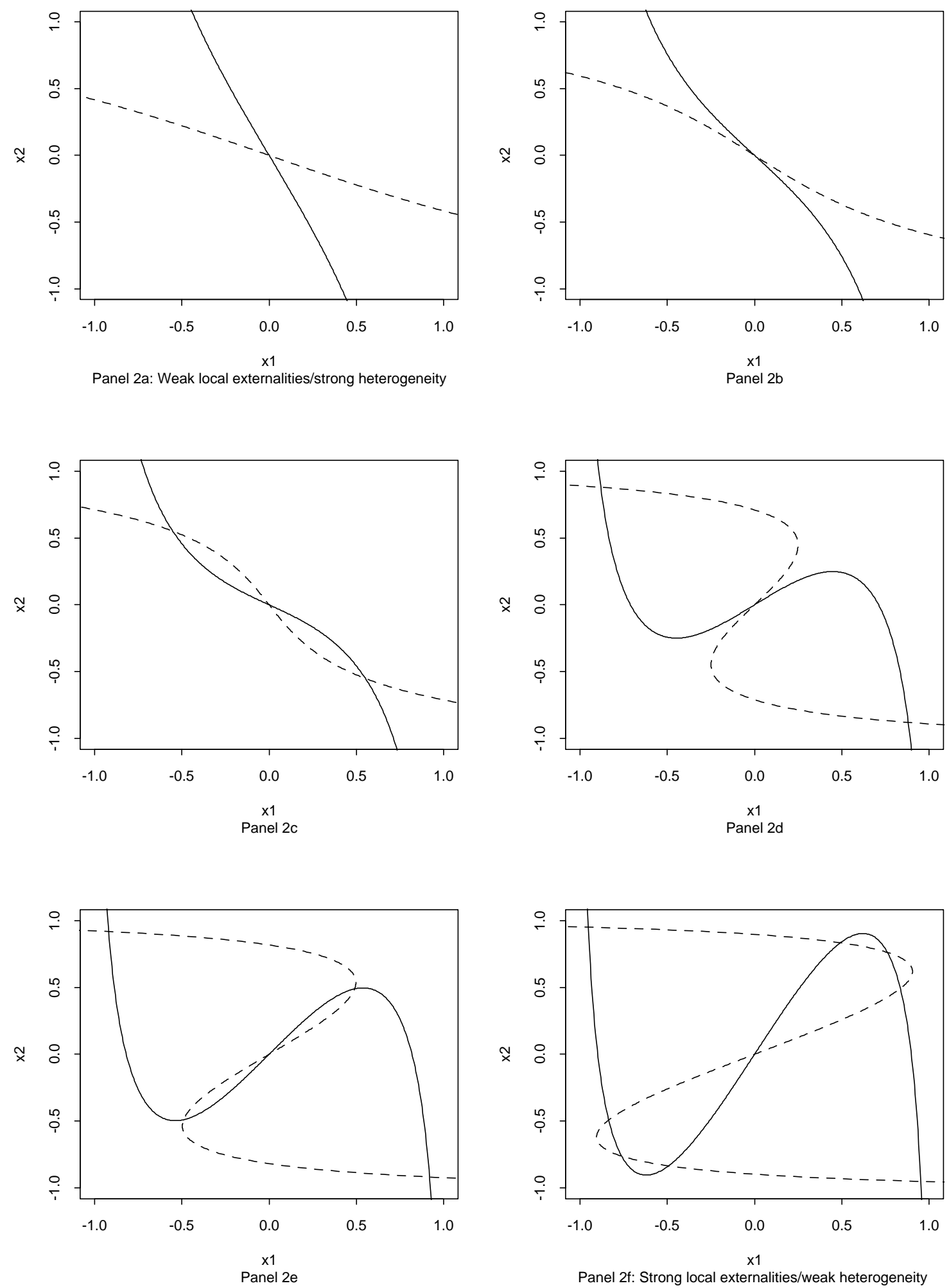

Equilibria exist at the intersections of the two curves.

The dashed curve is equation 6 ; the solid curve equation 7. 
Proportion using dominant technology



\section{References}

An, M. and N. Kiefer (1995), "Local Externalities and Societal Adoption of Technologies", Journal of Evolutionary Economics, vol 5: 103-117.

Anderson, S.P, A. dePalma and J. Thisse (1992). Discrete Choice Theory of Product Differentiation. Cambridge, Mass: MIT Press.

Arthur, W.B. (1989) "Increasing Returns, Competing Technologies and Lock-In by Historical Small Events: The Dynamics of Allocation under Increasing Returns to Scale", Economic Journal 99,116-131.

Bassanini, A. and G. Dosi (1998) "Competiting Technologies, International Diffusion and the Rate of Convergence to a Stable market structure", IIASA Interim Report \# IR-98012 .

Brock, W.A. (1993). "Pathways to Randomness in the Economy: Emergent Nonlinearity and Chaois in Economics and Finance", Estudios Econønomicos, vol 8(1): 3-55.

Brock, W. and S. Durlauf (1995). "Discrete Choice with Social Interactions I: Theory" Santa Fe Institute Working Paper \#95-10-084.

Conslick, J. (1996). "Why Bounded Rationality?", Journal of Economic Literature, 34: 669-700.

Cowan, R. and W. Cowan. (1994). "Local Externalities and Spatial Equilibria: On the Nature and Degree of Technological Standardization"; Research Report \#9421 Department of Economics, University of Western Ontario.

Cowan R. and W. Cowan, (1998) "On Clustering in the Location of R\&D: Statics and Dynamics" Economics of Innovation and New Technology, forthcoming.

David, P.A. (1985) "CLIO and the Economics of QWERTY", American Economic Review 75: $332-337$.

David P.A., D. Foray, and J-M. Dalle (1998). "Marshallian Externalities and the Emergence and Spatial Stability of Technological Enclaves", Economics of Innovation and New Technology, forthcoming.

David, Paul A. and Shane Greenstein (1990). "The Economics of Compatibility Standards: An Introduction to Recent Research", Economics of Innovation and New Technology vol. 1, Fall-Winter. pp 3-41.

Durlauf, S. (1993). "Nonergodic Economic Growth", Review of Economic Studies, 60, 349366.

Durlauf, S. (1997) "Statistical Mechanics Approaches to Socioeconomic Behavior" in The Economy as a Complex Evolving System II, W.B. Arthur, S.N. Durlauf and D. Lane, eds., Redwood City: Addison-Wesley.

Elder, K.R. and M. Grant, (1990). "Singular Perturbation Theory for Phase-Front Dynamics and Pattern Selection" Journal of Physics A23, L803.

Farrell, J. and G. Saloner, (1985). "Standardization, Compatibility and Innovation", Rand Journal of Economics, 16, 70-83.

Föllmer, H. (1974). "Random Economies with Many Interacting Agents", Journal of Mathematical Economics vol 1: 51-62. 
Foray, Dominique (1989). "Les Modeles de competition technologique: Une revue de la literature" in Revue d'Economie Industrielle No. 48.

Glaeser, E., B. Sacerdote and J. Scheinkman, (1996). "Crime and Social Interactions", Quarterly Journal of Economics, 111, 135-164.

Katz, M. and C. Shapiro (1985). "Network Externalities, Competition and Compatibility", American Economic Review, 75: 424-440.

Kiefer, N. and M. An, "Local Externalities and Societal Adoption of Technologies", Journal of Evolutionary Economics, vol. 5: 103-117.

Kiefer, N., Z. Ye and M. An (1991) "A Dynamic Model of Local and Global Interactions among Economic Agents", mimeo, Cornell University.

Kirman, A. (1997) "Economies with Interacting Agents" in W.B. Arthur, S. Durlauf and D. Lane (eds.) The Economy as an Evolving Complex System, II, Addison Wesley.

Metcalfe, S. (1998). Evolutionary Economics and Creative Destruction Routledge.

Pathria, R.K. (1972). Statistical Mechanics. Pergamon Press.

Revue d'Economie Industrielle (1992). Special Edition on Technological Variety, No. 52.

Schroedinger, E. (1952). Statistical Thermodynamics: A course of seminar lectures delivered in January-March 1944, at the School of Theoretical Physics, Dublin Institute for Advanced Studies 2d ed. Cambridge : Cambridge University Press

Thompson et al. (1992). "Neural Networks with Constrained Inputs as Models for Pattern Formation in Primate Visual Cortex" Journal of Biological Physics, vol. 18, pp. 217-245.

Turing, Alan. (1952). "The Chemical Basis of Morphogenesis", Philosophical Transactions of the Royal Society, Series B. Biological Sciences, vol. 237, pp. 37-72.

Waldram, J. R. (1985). The Theory of Thermodynamics Cambridge: Cambridge University Press.

Yellott, J.I (1977). "The Relationship between Luce's Choice Axiom, Thurstone's Theory of Comparative Judgement and the Double Exponential Distribution." Journal of Mathematical Psychology, vol. 15: 109-144. 


\section{Appendix}

In this appendix we give a derivation of the Boltzmann or Gibbs distribution which is invoked as the equilibrium condition in interacting agents models. We present the argument from which equations 6 and 7 arise. We will also remark on the double exponential distribution assumed in random utility models for the purpose of deriving the logistic distribution. ${ }^{19}$

Our derivation follows the technique of Schrodinger (1952) in which the distrbution flows effectively from combinatorial aspects of a system with large numbers of agents. This approach is sometimes referred to as the micro-cannonical approach. In this derivation, we begin with a system, or economy, containing a large but finite number of agents. The number of agents is sufficiently large that for an analyst to keep track of them all is in practice impossible (even if it might be possible in principle). Thus the analyst must make use of some statistical devices to make statements about macro variables if he is to maintain the position that micro-foundations are important.

Consider an economy with a large, finite number, $N$, of agents, and a finite, discrete choice set of $Q$ possible technologies. There are $n_{q}$ agents using technology $q$. We employ a random utility model so that the benefit to agent $i$ from using technology $q$ is $F(\cdot)+h_{i, q}$, where $h_{i, q}$ is a random variable distributed i.i.d. with zero mean for all agents. For the moment, the arguments of $F$ are unspecified. The aggregate benefits in the economy are $B=\sum_{i=1}^{N} b^{i}$. It is useful to disaggregate by technology rather than by agent: $B=\sum_{q=1}^{Q} n_{q} \overline{b_{q}}$, where $\overline{b_{q}}$ is the average benefit to users of technology $q$. Consider the vector $\mathbf{n}=\left\{n_{1}, n_{2}, \ldots, n_{q}, \ldots, n_{Q}\right\}$ of numbers of agents using each technology. Given that there are many agents, and that these agents are indistinguishable to the analyst, $\mathbf{n}$ can be produced by many different allocations of technologies to agents. This number is

$$
\Omega(\mathbf{n})=\frac{N !}{n_{1} ! n_{2} ! \ldots n_{Q} !}
$$

We can see that $\Omega(\mathbf{n})$ can be treated as a distribution of distributions. Pathria (1972, pp. 53-61) shows that in the limit as the number of agents gets large, "the mean values, the most probable values - in fact any values that occur with a nonvanishing probability-become identical!" Thus to analyse $\Omega$ we need only concern ourselves with its modal value. To find the modal value, we simply maximize, but first two constraints

19 See Cowan and Cowan (1995) for a more detailed presentation of the material contained here. 
must be observed. First, we must restrict the number of agents to $N$ : $\sum_{q} n_{q}=N$. Second, we momentarily fix the aggregate level of benefits. The economics of this assumption is as follows. The behaviour of agents in an economy is determined by fundamentals. If the fundamentals do not change, then even if the very minute details of which particular agent uses which technology are indeterminate, values of aggregate variables should be constant. Thus, if we are concerned with a particular economy, we should assume that its fundamentals determine outcomes of aggregate variables (which take no account of the identities of agents). Thus we impose the constraint $\sum_{q} n_{q} \overline{b_{q}}=B$. To maximize the $\log$ of $\Omega(\bar{n})$ we use the lagrangian:

$$
\mathcal{L}=\ln \left(\frac{N !}{\prod_{q} n_{q} !}\right)-\lambda\left(\sum n_{q}-N\right)-\beta\left(\sum n_{q} \overline{b_{q}}-B\right)
$$

Because $N$ is large we can use Stirling's approximation: $\ln x ! \approx x \ln x-x$, so

$$
\mathrm{d} \mathcal{L} / \mathrm{d} n_{q}=\ln n_{q}-\lambda-\beta \overline{b_{q}}=0 .
$$

Thus,

$$
n_{q}=\Lambda e^{\beta \overline{b_{q}}}
$$

Solving for $\Lambda$ yields

$$
n_{q}=\frac{e^{\beta \overline{b_{q}}}}{\sum_{q^{\prime}=1}^{Q} e^{\beta \overline{b_{q^{\prime}}}}}
$$

This equation describes the most likely distribution of technologies over agents. Other distributions are in principle possible, but if the number of agents is large other distributions can be safely ignored, as Pathria shows.

This is the Boltzmann distribution of technology use. The probability that an arbitrary agent will be using technology $q$ is proportional to the exponential of the average cost of using it. ${ }^{20}$ This distribution is familiar from discrete choice random utility models as the logistic distribution

\footnotetext{
20 There is here an implicit assumption that simply counting states is a good way of measuring the probability of their occurrance. That is, there is an assumption that any distribution $\mathbf{n}$ having aggregate benefit equal to $B$ is equally likely to occur. This assumption is used to make the result consistent with entropy maximzation. The assumption is in fact much weaker than it seems. If we are trying to model a situation in which it appears, on the face of it, that this assumption is broken, we can re-establish it by re-defining states. In effect, in our model we would clone the more likely states to create a group of similar states (in fact observationally identical states) all having the same probability. This will re-establish the equal probability assumption, and generates a model which is observationally equivalent to the world in which this cloning is not performed.
} 
It can be shown that there is a monotonic relationship between $\beta$ and the average $\bar{b}$ occupied by agents. Thus there is a monotonic relationship between $\beta$ and the value of the aggregate variable, $B$. Thus dependence of the probability of observing an agent receiving $b$, on the fundamentals which determine aggregate values is embedded in the $\beta$ in equation 2 .

The outstanding issue is the interpretation of $\beta$. If $\beta$ is zero, $n_{q}=1 / Q$. All technologies are equally common. If $\beta$ is large, $n_{q}$ goes to 0 or 1 -every agent uses the same technology, namely the one with the highest average benefits. These two results correspond to results that obtain if there is a large or small degree of agent heterogeneity. If agents' tasks are wildly different from each other, there will be little incentive to coordinate with neighbours, and each agent will 'do his own thing'. Since $h$ has zero mean, each technology will be equally prevalent. On the other hand, if all agents perform the same tasks, all will choose the same technology. ${ }^{21}$ Thus we can interpret $1 / \beta$ as being a measure of the degree of heterogeneity present in the system.

The derivation to this point is extremely general and can be (and has been) applied to many cases, based on many different benefit functions $F(\cdot)$. We now introduce the micro-economics of our model. The net benefit to an agent in regions $r$ from using technology $q$ is $g\left(Z_{r, q}\right)-f\left(Z_{q}\right)+h_{i, q}$ where $Z_{r, q}$ is the proportion of agents in region $r$ using $q, f\left(Z_{q}\right)$ is the price of $q$, determined by the total number of agents in the economy using $q$, and $h_{i, q}$ is the component idiosyncratic to the agent. Since the mean of $h$ is zero, $\overline{b_{q}}=g\left(Z_{r, q}\right)-f\left(Z_{q}\right)$.

$$
Z_{r, q}=\frac{e^{\beta\left(g\left(Z_{r, q}\right)-f\left(Z_{q}\right)\right)}}{\sum_{p=1}^{Q} e^{\beta\left(g\left(Z_{r, p}\right)-f\left(Z_{p}\right)\right)}} .
$$

One such equation exists for every region-technology pair, and finding equilibria consists in solving these equations simultaneously.

The Boltzmann distribution of actions over agents is a logistic distribution, familiar from discrete choice, random utility models. Yellott (1977) shows that to generate the logistic distribution for $x$ from a utility function of the form $U=f(x)+\epsilon$, that $\epsilon$ is distributed as a double exponential is both necessary and sufficient. This assumption is (implicitly) made, but seems rather ad hoc. The above derivation of the Boltzmann

21 This extends to the point that there is intertemporal, intra-agent heterogeneity by adding the proviso that each agent always performs the same task. 
distribution makes clear what is being brought in with this assumption. We can see that formally, in this derivation, the logistic distribution rests on three things: first, the assumption that counting states is an effective way of measuring probabilities; second, that the presence of many agents implies that combinatorial effects are important; third, that the fundamentals of the economy determine its arithmetic mean values. The first consideration implies that entropy is being maximized..$^{22}$ In practical terms, what this means is that an observation of a choice provides minimal information, after accounting for the deterministic choice factors, in predicting other choices - either those of other agents, or those of the same agent at a different time. In the Lagrangian, the second constraint is $\sum_{q} n_{q} \overline{b_{q}}=B$. This states that the fundamentals of the economy determine aggregate values of the variables, or equivalently, arithmetic means of them. If the fundamentals determined values other than arithmetic means, for example geometric means, or means of logarithms, the emergent distribution would have a different form. In the context of discrete choice models it seems reasonable that fundamentals, such as utility functions, prices or income, determine average behaviour. But this in turn implies that variables must be constructed and measured in such a way that this is the case.

22 See Brock (1993) for a further discussion of entropy maximzation in this context, and for a different discussion of the relationship between discrete choice models and interacting agents models. 